\title{
GPR and IRT tests in two historical buildings in Gravina in Puglia
}

\author{
Loredana Matera ${ }^{1}$, Raffaele Persico ${ }^{1}$, Edoardo Geraldi $^{1}$, Maria Sileo $^{1}$, and Salvatore Piro ${ }^{2}$ \\ ${ }^{1}$ Institute for Cultural and Monumental Heritage IBAM-CNR, Headquarter in Catania, Italy \\ ${ }^{2}$ Institute of Science for Knowledge, Conservation and Use of Cultural Heritage ITABC-CNR, Rome, Italy
}

Correspondence to: Raffaele Persico (r.persico@ibam.cnr.it)

Received: 28 April 2016 - Published in Geosci. Instrum. Method. Data Syst. Discuss.: 5 July 2016

Revised: 16 September 2016 - Accepted: 10 October 2016 - Published: 29 November 2016

\begin{abstract}
This paper describes a geophysical investigation conducted into two important churches, namely the Cathedral of Santa Maria Assunta and the Church of Santa Croce, both in Gravina in Puglia (close to Bari, southern Italy). The Church of Santa Croce, now deconsecrated, lies below the cathedral. Therefore, the two churches constitute a unique building body. Moreover, below the Church of Santa Croce there are several crypts, which are only partially known. The prospecting was performed both with a pulsed commercial ground penetrating radar (GPR) system and with a prototypal reconfigurable stepped frequency system. The aim was twofold, namely to gather information about the monument and to test the prototypal system. The GPR measurements have also been integrated with an infrared thermography (IRT) investigation performed on part of the vaulted ceiling in the Church of Santa Croce, in order to confirm or deny a possible interpretation of certain GPR results.
\end{abstract}

\section{Introduction}

Noninvasive diagnostic technologies, and in particular ground penetrating radar (GPR) and infrared thermography (IRT), are important tools to study architectural and monumental heritage. The use of non-destructive testing (NDT) arises from the exigency not to damage the probed structure, which is a particularly important requirement for cultural heritage. Noninvasive sensing makes it possible to gather both historical and structural information about the building at hand (Masini et al., 2010; Utsi, 2010; Calia et al., 2012; Grinzato et al., 2002; Geraldi et al., 2003, 2016; Carlomagno et al., 2011). In particular, it has been used to document the state of damage of masonries (Masini et al., 2010), columns and pillars (Binda et al., 2003; Leucci et al., 2011) or even statues (Kadioglu and Kadioglu, 2010; Sambuelli et al., 2011). NDT is also an important tool in detecting the presence of ancient tombs (Cardarelli et al., 2008), crypts, cavities (Piscitelli et al., 2007; Persico et al., 2014) and archaeological structures (Goodman and Piro, 2013), and is also a useful tool for the study of murals and frescoes (Pieraccini et al., 2006).

The GPR technique is based on the scattering of the electromagnetic wave radiated by a transmitting antenna and impinging on any buried anomalies, that are reflected along all the directions and in particular along the direction of the receiving antenna, which gathers a small share of this scattered energy. Depending on the characteristics of the medium and on the frequency of the antennae, the GPR technique can usually investigate the first metres of depth ( $\sim 1$ to $7 \mathrm{~m}$ ) with a resolution, depending on the characteristics of the medium and the frequency of the antenna, of the order of one half of the internal wavelength, which means of the order of 1$40 \mathrm{~cm}$. Usually, there is a degradation of the resolution vs. the depth.

The IRT technique (Maldague, 2001) is based on the thermal radiation and heat transfer mechanisms that occur between the target's surface and the thermal camera sensor (mainly emission of the target and reflection by the surroundings). The properties that lead to the thermo-physical phenomena (conduction and radiation) between the target, its surface and the surroundings (boundary conditions) are thermal properties, such as conductivity, diffusivity, effusion and specific heat capacity; spectral properties, such as emissivity, absorption, reflection and transmission and further physical properties, such as volumetric mass density, porosity and parameters defining the hygrometric conditions. An infrared camera measures the thermal radiation coming from the material under investigation and renders the image of the sur- 

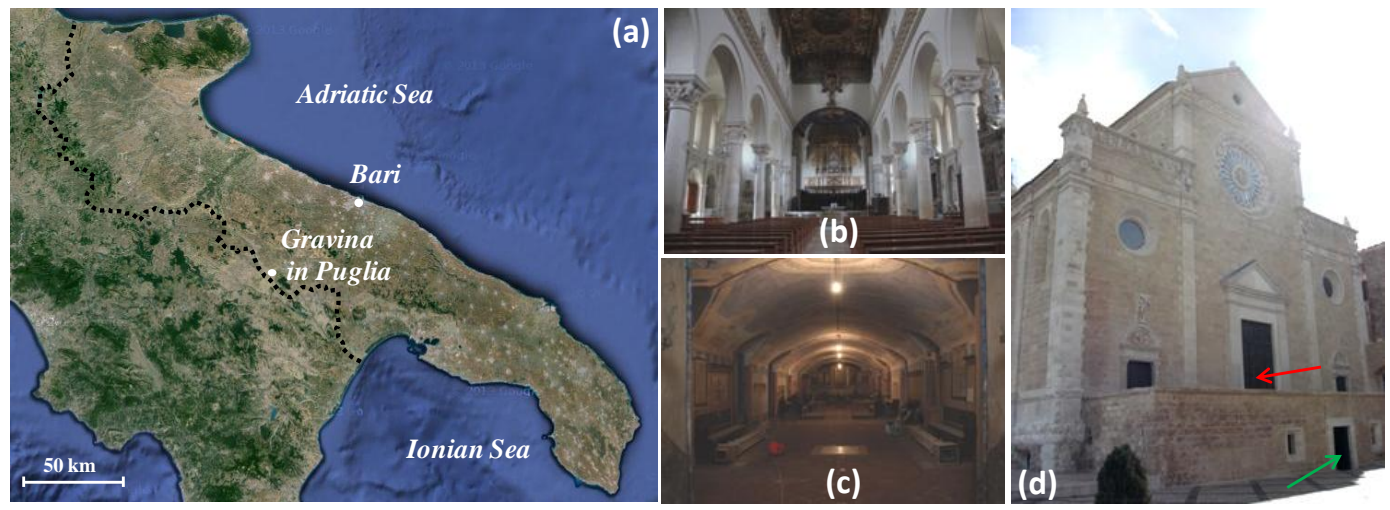

Figure 1. (a) Geographical location of Gravina in Puglia, (b) The central nave of the cathedral, (c) The central nave of the Church of Santa Croce, (d) The external façade of the building with the entrance of the cathedral (upper red arrow) and of the Church of Santa Croce (lower green arrow).

face area in relation to a temperature scale. The temperature mapping of the surface area is strictly connected to the ongoing thermal transient state generated actively from an exterior heat source or passively inducted from the variable environmental physical conditions (solar irradiation, wind, diurnal fluctuation of temperature, humidity, etc.) as well as from the medium physical characteristics (materials, texture, degradation processes, etc.).

In this paper, we present GPR and IRT results gathered in two churches in the town of Gravina in Puglia, on the outskirts of Bari (Apulia region, southern Italy), namely the Cathedral of Santa Maria Assunta and the underlying Church of Santa Croce.

In Fig. 1 the geographical location and images of the two churches are provided.

This building constitutes a natural test site for experimenting with the capabilities of a GPR system to penetrate into a multilayered structure. In particular, we will show how some of the main features of the Church of Santa Croce are reproduced from GPR data taken on the floor of the cathedral. We will also show images ascribable to a few crypts (some of which are accessible and some of which are not accessible) below the Church of Santa Croce, gathered from GPR data collected from the floor of Santa Croce.

Indeed, some historical sources (Lorusso et al., 2013) and some local rumours state the presence of at least two levels below the Church of Santa Croce, and even a third level is hypothesised.

However, the reflection of the electromagnetic waves from a large cavity is generally quite strong, and customarily it is quite hard to identify a second (large) cavity piled below a shallower (also large) one because the shallower cavity can mask the deeper one. With regard to the case history at hand, we were unable to identify the crypts under the Church of Santa Croce from the floor of the upper-lying cathedral, and therefore infer that we were also unable to identify levels further below the crypts identified from the floor of Santa Croce.
The prospecting was performed with two different GPR systems, namely a pulsed Ris Hi-mode system, manufactured by IDS Corporation, equipped with a double antenna at 200 and $600 \mathrm{MHz}$ and a prototypal stepped frequency reconfigurable system (Persico and Prisco, 2008). The prototypal system was implemented within the research project AITECH (http://www.aitechnet.com/ibam.html) by the Institute for Archaeological and Monumental Heritage, National Research Council (IBAM-CNR) together with the University of Florence and the IDS Corporation, and underwent several tests in different situations within a Ph.D. course handled in collaboration between the University of Bari and the Institute for Archaeological and Monumental Heritage, National Research Council (IBAM-CNR) (Persico et al., 2014).

Finally, after examining the GPR results, we deemed it worth performing another noninvasive test with an infrared camera on part of an internal wall of the cylindrically vaulted ceiling of the main nave of the Church of Santa Croce, in order to collect further elements for a more reliable interpretation of the GPR data.

The paper is organised as follows: in the next section a brief historical description of the two monuments is provided, in Sect. 3 a brief description of the reconfigurable system is given, in Sect. 4 the GPR measurement campaigns are described and the main results are shown, in Sect. 5 the infrared investigations are addressed and conclusions follow in Sect. 6.

\section{Historical outlook}

Historical sources (D'Elia, 1975) report that the presence of two overlapping or multilayered churches, as in the case of the Cathedral of Santa Maria Assunta and the Church of Santa Croce in Gravina in Puglia, is common in several Apulian churches (e.g. in the towns of Bari, Trani and Bisceglie). The most probable reason for such architecture is that there 


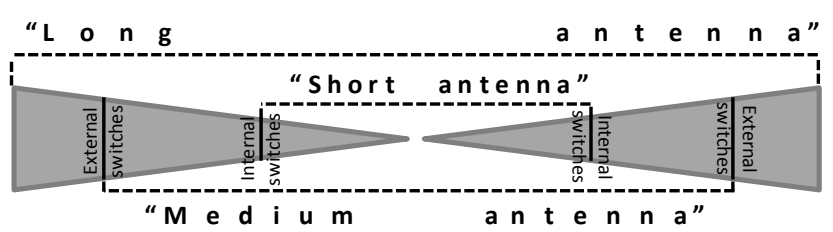

Figure 2. Non-quantitative scheme for the reconfigurable antennae: the antennae are "short" if all the switches are set off, they are "medium" if the internal switches are set on and the external ones are set off, they are "long" if all the switches are set on.

was an earlier church incorporated in the upper-lying one because the demolition of the earlier church was discouraged due to the sacredness of the place.

With regard to the building at hand, the construction of the Cathedral of Santa Maria Assunta, in Apulia-Romanesque style, dates back to 1092 (Gelao, 2005).

During the centuries, it has been reconstructed with many difficulties and interruptions due to scarcity of funds and several earthquakes that afflicted the town of Gravina. Only a few parts of the ancient cathedral (the rose window, some external frames and some capitals of the arches that lead into the presbytery from the aisles) have been preserved.

Some authors (Gelao, 2005) report that the Cathedral of Santa Maria Assunta was built over the Church of Santa Croce in order to overcome difficulties related to the topographic morphology of the site, which would have compelled the initial constructors to build the Church of Santa Croce within a natural depression, locally called a gravina.

Today, the cathedral has two entrances, one of which is located on the southern façade in Benedetto XIII Square, whereas the other entrance (the portal shown in Fig. 1d) is located in the main façade with a few stairs above ground level in front of a rectangular open space.

A big chapel protrudes from the left hand aisle and stands sheer on a deep gravina. This big chapel was built in the first half of the 17th century with two floors. The oratory is on the lower floor in the Church of Santa Croce. The Blessed Sacrament Chapel is on the upper floor in the cathedral. A further prestigious architectural element is the bell tower (Lorusso et al., 2013).

Inside the cathedral there are three naves, separated from each other by 14 columns linked together by round arches. The ceiling of the nave is wooden, carved and gilded according to the Baroque style. The entire cathedral covers an area of $50 \times 20 \mathrm{~m}^{2}$. The height of the nave is $20.90 \mathrm{~m}$, while that of the aisles is $12.70 \mathrm{~m}$. More detailed information about the cathedral can be found in Lorusso et al. (2013).

The underlying Church of Santa Croce is also constituted of a central nave and two lateral aisles, divided by massive squared pillars with barrel vaults, and has three altars. The church was closed to worship in 1958, and until then had been used to bury corpses. Therefore, graves were dug underneath the floor of the church (Lorusso et al., 2013). Today, some frescoes and 12 graves, dating back to a period from the 16th to the 19th century, remain (Lorusso et al., 2013).

\section{The prototypal stepped frequency system}

The prototypal reconfigurable system used in the case history at hand is a stepped frequency GPR, whose frequency range extends from $50 \mathrm{MHz}$ to $1 \mathrm{GHz}$. This range can be swept with a frequency step optionally equal to 5 or $2.5 \mathrm{MHz}$. One of the main innovation points related to this system is that it is equipped with three couples of equivalent antennae. These three couples are created from a unique physical couple of antennae by means of two series of electronic switches (implemented by means of PIN diodes) displaced along the arms of the two antennae according to the scheme of Fig. 2. The three couples of equivalent antennae are achieved by switching the PIN diodes on and off, which provides a couple of "long antennae" if both switches per arm are set on, a couple of "medium antennae" if the external switches are set off and the internal switches are set on and "short antennae" if both switches are set off. In other words, the switches implement two subsequent equivalent cuts of the arms of the antennae. We have seen that (with some site dependent variations) the central frequencies are of the order of 120,250 and $550 \mathrm{MHz}$ for the long, medium and short antennae respectively. Moreover, as is usual with GPR antennae, the bands are of the same order as the central frequencies.

Indeed, the problem of the cut of the arms is more complicated than it might seem because the detached parts of the arm provide contributions both to the antenna pattern and to the input impedance, since some induced currents flow on them. However, from a practical point of view, there are two factors that mitigate the problem. The first factor is that the antennae are bow tie, and this makes the axis of the arms a direction of null of the pattern. Therefore, the parasitic current developed on the (collinear) detached parts is expected to be tolerable. Stronger induced currents might develop instead if the detached parts were parallel to the active part of the antennae, as it happens in the case of a Yagi-Uda antenna (Grajek et al., 2004). In particular, the only component of the field that might induce meaningful currents on the detached parts in our case is the radial one, which vanishes proportionally to the inverse of the square of the distance from the gap (unlike the longitudinal component, which attenuates as the inverse of the distance). A second factor that mitigates the effect of the cuts is that, when the short antennae radiate and receive the signal, the cut part of the arms is in turn cut into two parts, which prevents the development of two strong parasitic currents.

In the case history at hand, we gathered the entire available range of frequencies, $50-1000 \mathrm{MHz}$, with each of the three equivalent antennae, and then filtered the data relative to each antenna into their own bands. 


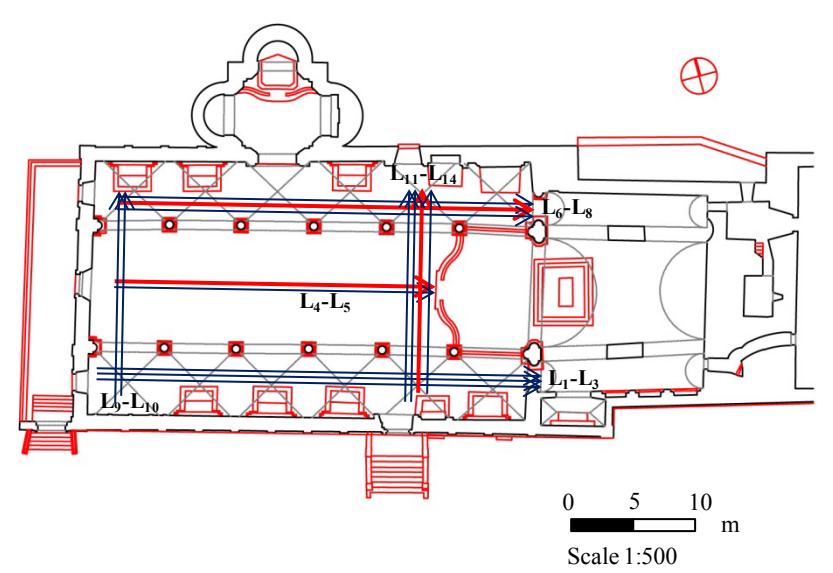

Figure 3. Map of the Cathedral of Santa Maria Assunta with the layout of the gathered B-scans.

Incidentally, the prototypal system allows the reconfiguration of not only the length of the arms of the antennae but also the radiated power, and above all, the integration time (Noon, 1996) for each radiated harmonic signal. However, these two options are not of interest with regard to the case history at hand and will not be further mentioned here. For the interested reader, more details about the reconfiguration of the integration times are provided in Persico et al. (2016).

\section{GPR results}

\subsection{Results in the cathedral}

The layout of the B-scans gathered in the Cathedral of Santa Maria Assunta is shown in Fig. 3. A total of 14 B-scans $\left(\mathrm{L}_{1}-\right.$ $\mathrm{L}_{14}$ ) were acquired, eight of which are parallel to the main nave and six of which are orthogonal to it. The length of the six B-scans recorded in the two aisles ranged from 30 to about $32 \mathrm{~m}$, namely the $\mathrm{B}$-scans $\mathrm{L}_{1}-\mathrm{L}_{3}$ in the right hand aisle and the $\mathrm{B}$-scans $\mathrm{L}_{6}-\mathrm{L}_{8}$ in the left hand aisle. The length of the B-scans in the main nave $\left(\mathrm{L}_{4}\right.$ and $\left.\mathrm{L}_{5}\right)$ was about $24 \mathrm{~m}$. The length of the B-scans $\mathrm{L}_{9}-\mathrm{L}_{10}$, close to the entrance of the cathedral, was about $14 \mathrm{~m}$ and the length of the B-scans close to the transept $\left(\mathrm{L}_{11}-\mathrm{L}_{14}\right)$ was about $18 \mathrm{~m}$ long. The church currently is in use and we did not have permission for gathering a complete grid of data. Moreover, we have represented the projection of the $\mathrm{B}$-scans $\mathrm{L}_{4}, \mathrm{~L}_{7}$ and $\mathrm{L}_{13}$ on the map of the underlying Church of Santa Croce shown in Fig. 4. This will make it easier to understand the following part of this section. We have created this representation from the quantitative maps of the two churches, which can be precisely "hanged" to each other through the above-described chapel protruding on the gravina.

Three of the B-scans, gathered with both the commercial and the prototypal systems, are shown in Figs. 5 and 6. The data were processed with the ReflexW code (Sand-

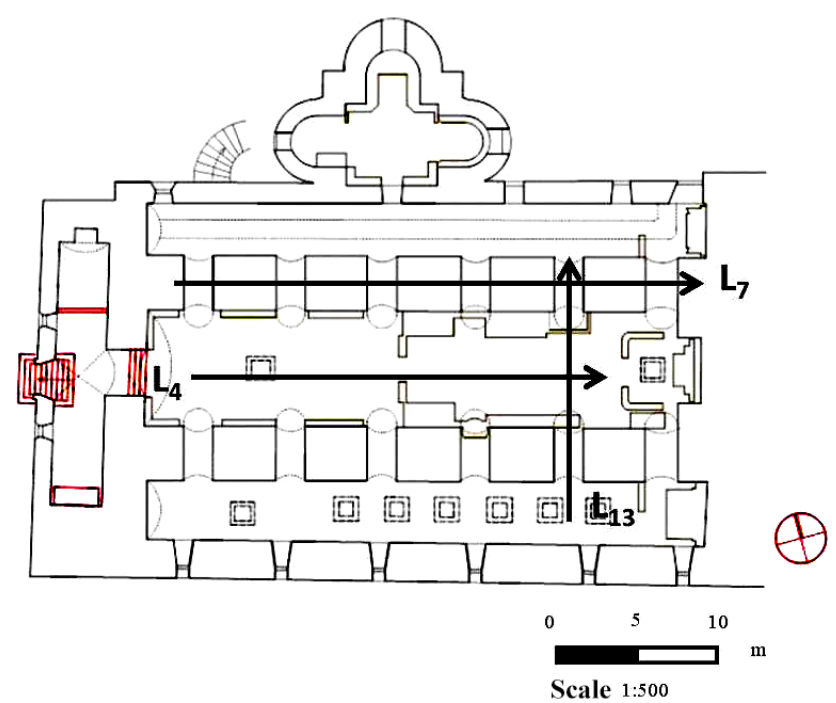

Figure 4. Map of the Church of Santa Croce, with the projections of the layouts of the B-scans $\mathrm{L}_{4}, \mathrm{~L}_{7}$ and $\mathrm{L}_{13}$ gathered on the floor of the Cathedral of Santa Maria Assunta.

meier, 2011) through the following steps: zero timing, twodimensional filtering constituted by a subtracting average on 40 traces, variable gain vs. the depth and a further onedimensional bandpass filtering. For the collected data, no migration was carried out, because the anomalies looked for were indeed quite large, and this focusing procedure did not provide satisfying results. This is probably due to the meaningful nonlinear effects related to the size and complexity of the scattering anomalies at hand (Persico et al., 2002).

The radar sections reported in the upper panels of Figs. 5 and 6 (which refer to the B-scan $\mathrm{L}_{7}$, evidenced by a red arrow in Fig. 3 and a black arrow in Fig. 4) were created in the right hand aisle (looking toward the altar) and refer to the data gathered with the pulsed and prototypal systems respectively. The recorded signals at 14 ns (see the yellow arrows in Figs. 5 and 6) indicate the presence of six single arches at the same time-depth and equally spaced at about $5.6 \mathrm{~m}$ from each other. Another signal at $40 \mathrm{~ns}$ (see the yellow arrows in Figs. 5 and 6) is also present, even more visible than the reflections at $14 \mathrm{~ns}$. The first signals at $14 \mathrm{~ns}$ are related to the top of the vaults of the over-crossed part of the ceiling of the Church of Santa Croce. In particular, the six arches correspond to the underlying arches of the lateral openings between the central nave and the lateral aisles of Santa Croce, as can be understood from Fig. 4.

From the diffraction hyperbolas, we have evaluated a propagation velocity of the electromagnetic waves about equal to $0.12 \mathrm{~m} \mathrm{~ns}^{-1}$. Therefore, the upper surface of the six vaulted features is estimated to be at about $0.85 \mathrm{~m}$ from the floor of the cathedral, while the thickness of the structure is estimated to be about $4 \mathrm{~m}$, in good agreement with the ground truth visible from the Church of Santa Croce. Moreover, in B-scan L7 

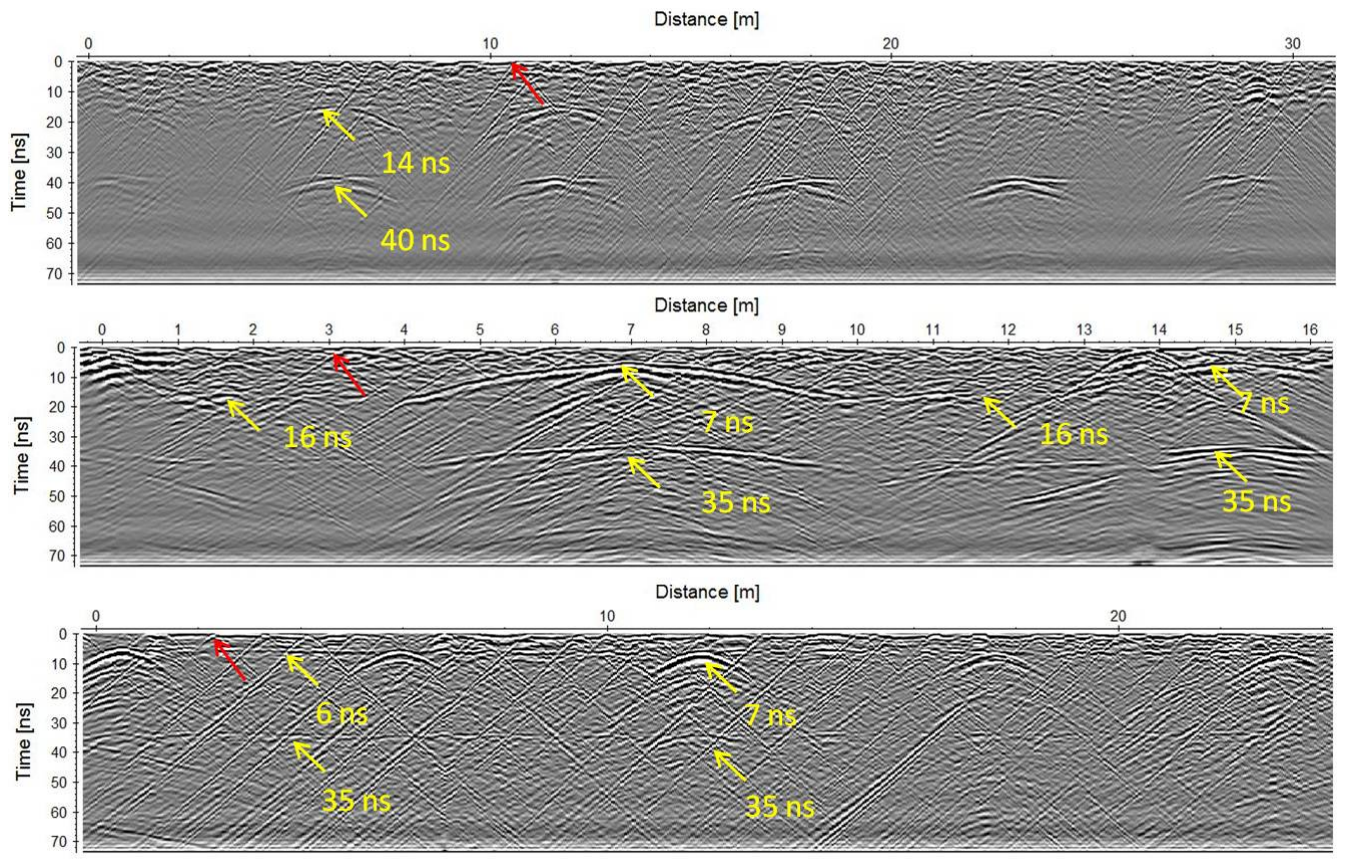

Figure 5. Radar sections recorded with the antennae at $600 \mathrm{MHz}$ of the commercial system in the cathedral: $\mathrm{L}_{7}$ (upper panel), $\mathrm{L}_{13}$ (middle panel), $\mathrm{L}_{4}$ (lower panel).
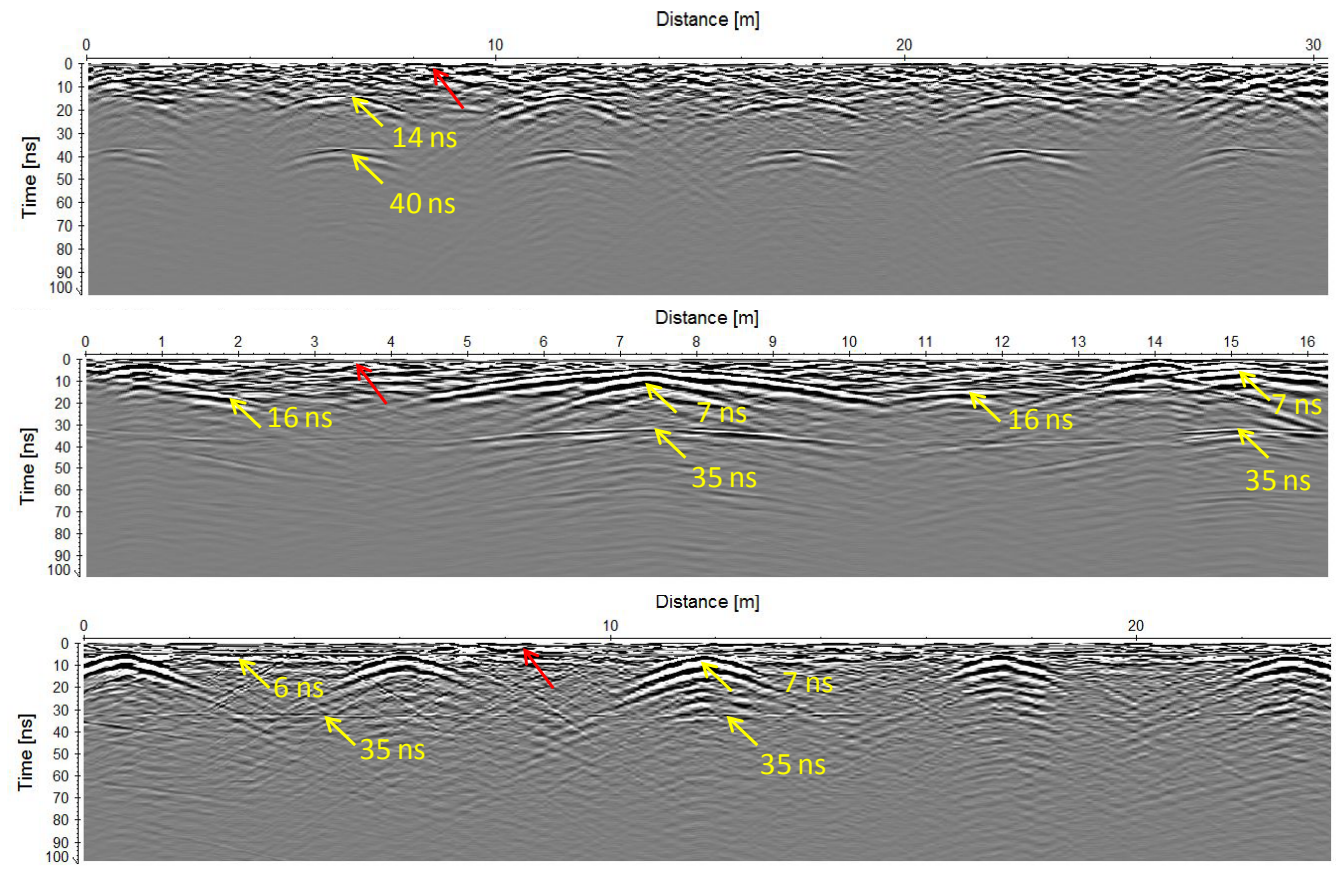

Figure 6. Radar sections recorded with the "medium antennae" of the GPR-R prototype system in the cathedral: $\mathrm{L}_{7}$ (upper panel), $\mathrm{L}_{13}$ (middle panel), $\mathrm{L}_{4}$ (lower panel).

(but even more in the subsequent image of B-scan $\mathrm{L}_{4}$ ) very superficial signals equally spaced are also present (red arrows in Figs. 5 and 6). They are likely ascribable to a welded steel mesh (with a step of about $50 \mathrm{~cm}$ ) under the floor of the cathedral (that of course is not original). Below the time depth of $40 \mathrm{~ns}$, multiple signals are also recorded.

The B-scans shown in the middle panels of Figs. 5 and 6 have been acquired in the transept of the cathedral $\left(\mathrm{L}_{13}\right.$, 
marked with a red arrow in Fig. 3 and evidenced also in Fig. 4). In both radar sections it is possible to notice the presence of a first flat ceiling placed at the time-depth of $16 \mathrm{~ns}$, about $3 \mathrm{~m}$ long, followed by a vaulted ceiling whose top is at $7 \mathrm{~ns}$, about $6 \mathrm{~m}$ long, followed by a flat ceiling again at $16 \mathrm{~ns}$, about $4 \mathrm{~m}$ long, followed by a further vaulted ceiling whose top is at $7 \mathrm{~ns}$, about $2 \mathrm{~m}$ long. According to the evaluated propagation velocity, the depth of the top of the vaults appears to be of the order of $0.40 \mathrm{~m}$ (but is probably slightly larger for engineering reasons) under the floor of the cathedral, while the two flat ceilings appear to be at a depth of about $1 \mathrm{~m}$. Under this "comprehensive" curved-line-shaped reflector, there is a flattish reflector at the depth of about $35 \mathrm{~ns}$ that we interpret as the reflection from the floor of the Church of Santa Croce. Making use of the estimated propagation velocity of $0.12 \mathrm{~m} \mathrm{~ns}^{-1}$ before the curved-line-shaped reflector, and making use of the propagation velocity in free space beyond it, we estimate a thickness of $2.85 \mathrm{~m}$ under the two flat ceilings and a maximum height of the underlying room of about $4.20 \mathrm{~m}$ below the top of the vaulted reflector. This is coherent with the height of the underlying Church of Santa Croce in the main nave and the lateral aisles, as well as with the height of the corridors that connect them to each other. In this B-scan further buried structures are also recorded. In particular, similar to the previous B-scan, some superficial signals (red arrows in the middle panels of Figs. 5 and 6) and multiple signals below $35 \mathrm{~ns}$ are observed.

The third image, reported in the lower panels of Figs. 5 and 6 is B-scan $\mathrm{L}_{4}$, taken in the nave of the cathedral, marked with a red arrow in Fig. 3 and also evidenced in Fig. 4. Two flat reflections throughout the length of the radar sections at a time-depth of $6 \mathrm{~ns}$ and of $35 \mathrm{~ns}$ are visible (they are indicated with yellow arrows in the lower panels of Figs. 5 and 6). We estimate that these signals are ascribable to the ceiling and floor of the underlying church nave. In this image, the ceiling of the main nave of the Church of Santa Croce appears flat because the GPR path is parallel to the main nave. The time depth of $6 \mathrm{~ns}$ (quite close to the top of the vaults at $7 \mathrm{~ns}$, visible in the previous panel) indicates that we have passed quite close to the top of the underlying cylindrically vaulted ceiling. In the lower panels of Figs. 5 and 6, five hyperbolic reflectors are also visible, with their tops at about $7 \mathrm{~ns}$.

The hyperbolic reflectors are indicated with further yellow arrows in Figs. 5 and 6. The reflectors are spaced about $5.75 \mathrm{~m}$ apart from each other, starting from the abscissas $0.5 \mathrm{~m}$. We interpret these reflectors as reinforcement structures, developing in the direction orthogonal to the nave, or keystones made with a different material than the surrounding bricks.

In this radar section the contribution of some shallow metallic object and some signal, probably arising from multiple reflections beyond the time depth of $40 \mathrm{~ns}$, is visible.

In summary, the B-scans shown in Figs. 5 and 6 show that the two GPR systems provide results in good agreement with each other. The main difference regards the contribution of

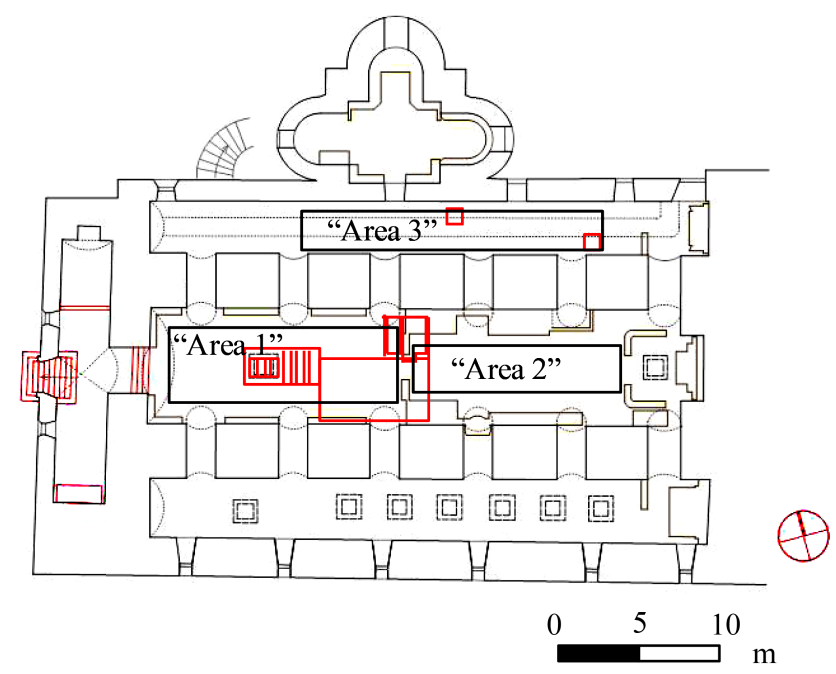

Figure 7. Map of the Church of Santa Croce with the three investigated areas, with the map of the underlying crypt and with two manholes evidenced in red contour.

the superficial welded steel mesh, which appears less marked in the prototype radar sections.

\subsection{Results in the Church of Santa Croce}

In the Church of Santa Croce, three different areas, marked in Fig. 7 with black rectangles and labelled "Area1", "Area2" and "Area3", have been investigated. In the plan of Fig. 7, a known hypogeal chamber tomb under the floor is represented by means of red contours. The chamber tomb is composed of a main rectangular room and a lateral niche used for sepultures, connected to the floor of Santa Croce with a straight staircase that leads to a marble manhole.

Other manholes are also visible in the lateral aisles, but only the manhole relative to the represented chamber is accessible and could be opened. For each area, an orthogonal grid of B-scans with an interline step of $50 \mathrm{~cm}$ was gathered with both the GPR systems at hand. Likewise, the data gathered in the cathedral, and the data gathered in Santa Croce were processed using the ReflexW code.

The data of Santa Croce were migrated in time domain, making use of a propagation velocity of the electromagnetic waves equal to $0.09 \mathrm{~m} \mathrm{~ns}^{-1}$, and retrieved on the basis of the diffraction hyperbolas.

From the processed B-scans, horizontal depth-time slices from 0 to $70 \mathrm{~ns}$ were retrieved, with time windows of $\Delta t=$ $5.5 \mathrm{~ns}$. The most significant depth-time slices for both the GPR systems have been reported in Fig. 8. They refer to data collected with the antennae at $600 \mathrm{MHz}$ for the pulsed system and with the "medium antennae" for the GPR reconfigurable prototype system.

As it can be seen, the two GPR systems are in good agreement with each other, but the GPR reconfigurable prototype 

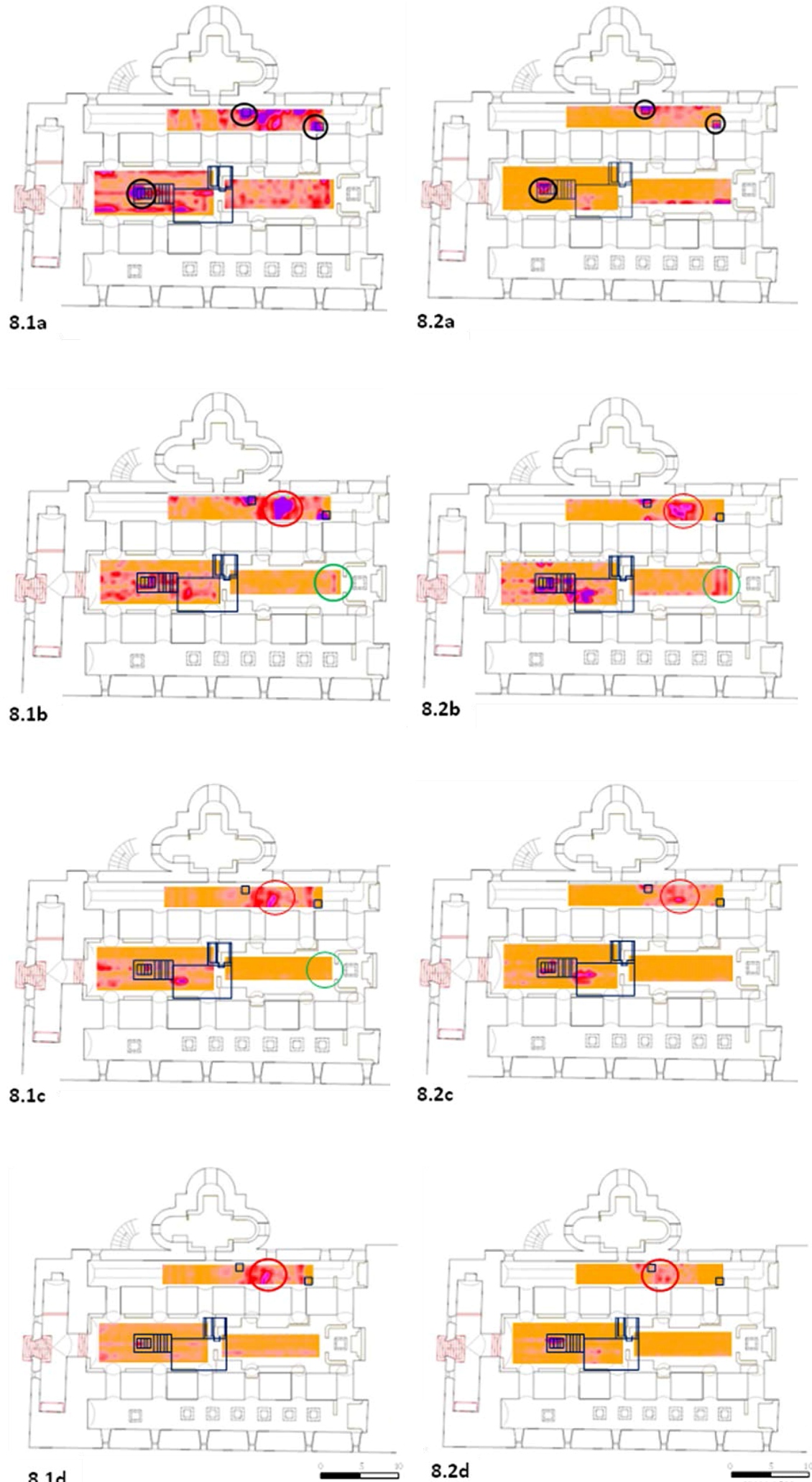

8.1d

Figure 8. A comparison of the results achieved with the antennae at $600 \mathrm{MHz}$ for the pulsed system (Fig. 8.1a-8.1d) and the "medium antennae" for the GPR-R prototype system (Fig. 8.2a-8.2d). 
depth-time slices provide better localised anomalies than those obtained with the pulsed GPR device.

With regard to Area 1, the prospected area covers a surface of $61 \mathrm{~m}^{2}$, which corresponds to 38 B-scans in all, 10 of which are parallel to the nave and 28 of which are orthogonal to it. In Area 2, 30 B-scans were recorded, covering an area of $29 \mathrm{~m}^{2}$. With regard to Area 3, in the right hand aisle, the surveyed area was about $33 \mathrm{~m}^{2}$ (5 B-scans parallel to the aisle and 34 orthogonal to it). It was not possible to prospect the entire aisle because of irremovable obstacles.

From the first depth-time slice at $5 \mathrm{~ns}$, corresponding to about $22 \mathrm{~cm}$ (Fig. 8.1a-8.2a) we see that both systems enounce the presence of shallow localised anomalies, which are three manholes. At the depth of $15 \mathrm{~ns}$ (about $70 \mathrm{~cm}$ ) a strong and large anomaly is identified in Area 3, probably corresponding to a chamber tomb whose ancient entrance was through the closest manhole on the left hand side of the spot in Area 3. Still, at $15 \mathrm{~ns}$ a strong anomaly in Area 1 is identified. It is probably ascribable to the ceiling of the large underlying chamber tomb. However, the spot has an extension significantly reduced with respect to the actual extension of the prospected area over the chamber. This is partially explained by the fact that the ceiling of the underlying chamber is not flat. In particular, there is a cylindrically vaulted ceiling in the first part of the chamber (i.e. the left hand part, closest to the steps that lead to the manhole in the map in Fig. 7) and a second part of the room where the ceiling is more than one metre deeper. At the depth of about $35 \mathrm{~ns}$, again two meaningful anomalies from Area 1 and Area 3 are visible. They might correspond to the floors of the two chambers.

With regard to Area 2, the main identified anomaly is indicated with a green circle in Fig. 8.1b and 8.2b. We interpret it as another possible tomb, smaller than the first one, whose entrance may be the manhole on the final altar visible in the plans in Figs. 7 and 8.

\section{IRT results}

Beyond the features visible from the B-scans and the depth slices shown in the previous section, there was some concern in regard to several longitudinal B-scans gathered along the short side of the main nave of the Church of Santa Croce. In particular, with reference to Fig. 9, we can see a central target at the time depth of about $30 \mathrm{~ns}$ that might be misinterpreted as the cross point of two large diffraction hyperbolas, or the top of a buried wall. In fact, this anomaly is visible along all the B-scans performed along the direction orthogonal to the main nave of the church (Areas 1 and 2), and it is equally visible with both systems.

Indeed, there is a central sequence of old lights hung from the ceiling. However, even if the lights were possibly the cause of the feature in Fig. 9, the shape of the anomaly was quite different from a diffraction hyperbola. Therefore, we have investigated the presence of further possible long

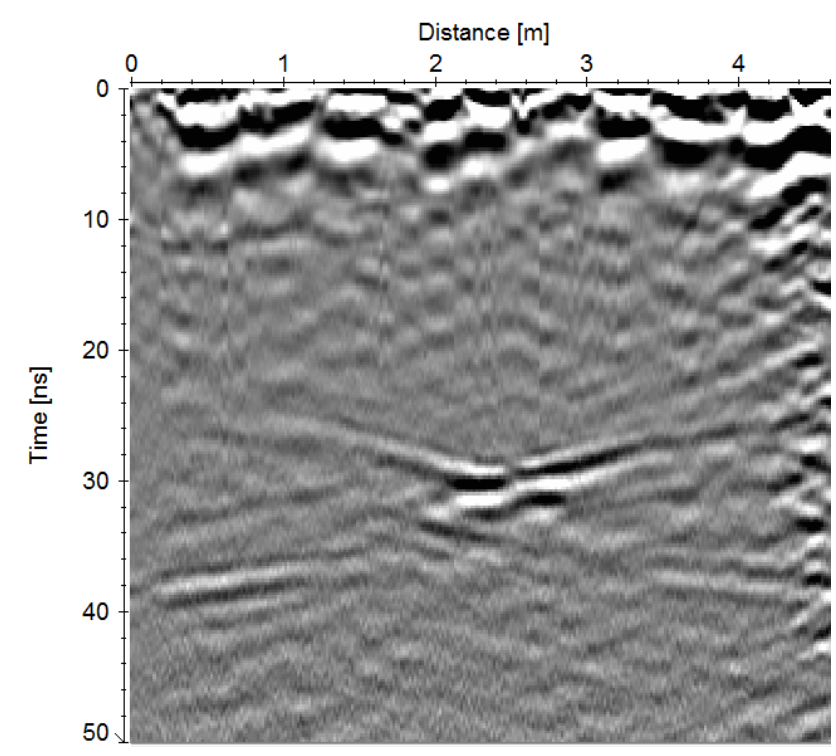

Figure 9. B-scan along the short side of the main nave of the Church of Santa Croce.

targets inserted in the wall (e.g. internal pipes or internal electrical wires). To do this, the vaulted ceiling was noninvasively probed with a FLIR SC 660 infrared camera (focal plane array detector, uncooled microbolometer, operating in the spectral range between 7.5 and $13 \mu \mathrm{m}$ ). The initial surface temperature of the segmental barrel vault was between 10.2 and $10.9^{\circ} \mathrm{C}$, the surface temperature of the floor of the room was $9.2^{\circ} \mathrm{C}$ and the air temperature was around $15^{\circ} \mathrm{C}$ (this last value was probably originally lower and was influenced from the opening of the church). In order to perform this measurement properly, the areas to be investigated were preventively heated for 60 min with $2 \times 500 \mathrm{~W}$ halogen lamps. The geometrical shape of the vault as well as entity and duration of the thermal stress had to be considered due to the presence of frescoes on the vaults. To preserve the integrity of the frescoes the lamps were kept around $2.5 \mathrm{~m}$ from the surface to be investigated, producing an increase of vault surface temperature of around $3.5^{\circ} \mathrm{C}$. As a consequence, the thickness of the transient state inducted was limited to the first millimetres of the medium. This statement is supported by some considerations on the physical properties of the constituent materials, in particular thermal diffusivity. However, this was not a major drawback in the case at hand because the source of the anomaly, if any, was expected to be superficial. The recording, tested every $120 \mathrm{~s}$ at the beginning and end of the heating, showed the best contrast during the last $360 \mathrm{~s}$ of the heating phase. From the thermal images shown in Fig. 10, we can appreciate anomalies mainly ascribable to the discontinuities of the ceiling frescoes' emissivity, in turn due to the different pigments that characterise the frescoes (see panels c and d). Further weaker anomalies seem to depend on the shallower level of the ashlars of stone below the plaster (see 


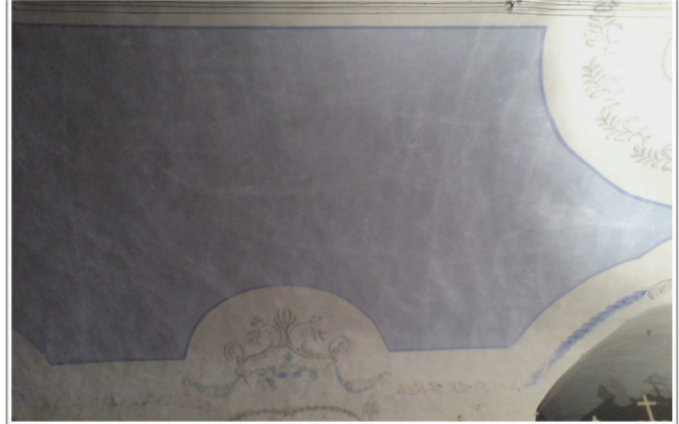

(a)

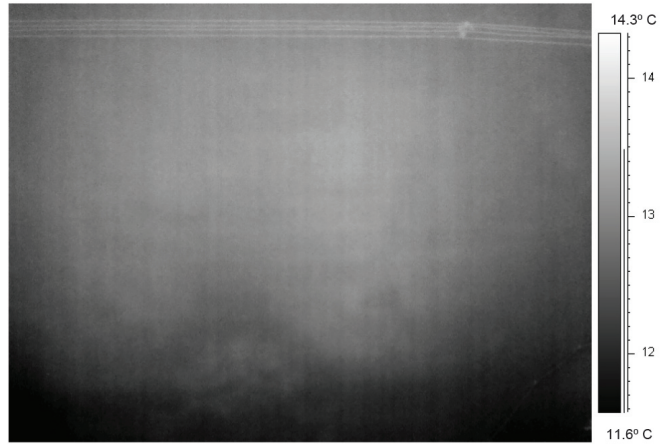

(c)

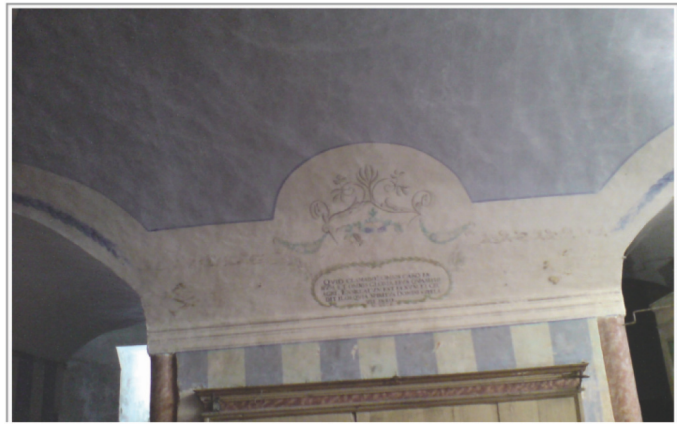

(b)

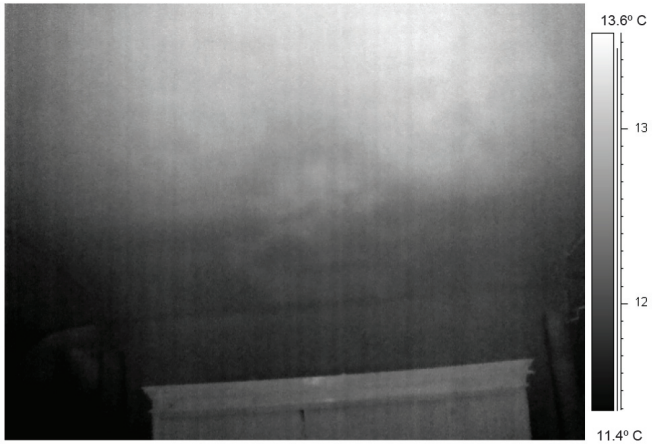

(d)

Figure 10. The IRT investigated areas in the nave of the Church of Santa Croce (panel a and panel b), IRT images relative to the photos of panels (a) and (b) (panel $\mathbf{c}$ and panel d).

panel $\mathrm{c}$ along the directrix of the vault). In particular, the IRT images do not show any evidence of internal pipes or wires, excluding such a hypothesis.

\section{Conclusions}

In this paper, the results of a non-destructive survey performed inside the Cathedral of Santa Maria Assunta and the Church of Santa Croce in Gravina in Puglia (Apulia, Italy) have been shown. We tested, in particular, an innovative stepped frequency GPR system vs. a traditional one, and have shown that it provided results fully comparable with those achieved with a traditional GPR system. An advantage of the prototype is that there are more bands at disposal to look for the best imaging of the targets of interest. This survey allowed us to verify that the main features of the Church of Santa Croce are visible from the upper floor of the Cathedral of Santa Maria Assunta, and allowed us to identify the presence of underlying structures below the Church of Santa Croce. However, neither the pulsed system nor the prototypal stepped frequency system was able to see chambers below the first buried level under the observation line. Therefore, the question of whether further levels are present underneath the crypts below the Church of Santa Croce remains open.

\section{Data availability}

Both GPR and IRT data relative to this paper are available in the online repository CNRSOLAR http://eprints.bice.rm.cnr. it/15594/.

Acknowledgements. The project AITECH, that allowed the implementation of the reconfigurable GPR system, was financed by the Apulia Region.

Edited by: J. Dumoulin

Reviewed by: three anonymous referees

\section{References}

Binda, L., Saisi, A., and Tiraboschi, C.: Application of sonic and radar tests on the piers and walls of the Cathedral of Noto, Constr. Build. Mater., 17, 613-627, doi:10.1016/S0950-0618(03)000564, 2003.

Calia, A., Leucci, G., Nicola, M., Matera, L., Persico, R., and Sileo, M.: Integrated prospecting in the crypt of the Basilica of Saint Nicholas in Bari, Italy, J. Geophys. Eng., 9, 1-11, 2012.

Cardarelli, E., Fischanger, F., and Piro, S.: Integrated geophysical survey to detect buried structures for archaeological prospecting. A case-history at Sabine Necropolis (Rome, Italy), Near Surf. Geophys., 6, 15-20, 2008. 
Carlomagno, G. M., Di Maio, R., Fedi, M., and Meola, C.: Integration of infrared thermography and high-frequency electromagnetic methods in archaeological surveys, J. Geophys. Eng., 8, S93 pp., 2011.

D’Elia, P. B.: Alle sorgenti del romanico Puglia XI secolo, seconda edizione (1987), Edizioni Dedalo, Pagine 344, ISBN: 978-88220-4107-4, 1975.

Gelao, C.: Puglia rinascimentale, Collana Patrimonio artistico italiano, Editore Jaca Book, Milano, p. 318, ISBN: 88-16-60343-7, 2005.

Geraldi, E., Gizzi, F. T., and Masini, N.: Termografia all'infrarosso ed archeologia dell' architettura: alcuni esempi. $22^{\circ}$ Convegno G.N.G.T.S., Sessione geofisica applicata ai Beni Culturali, 373375, November 2003.

Geraldi, E., Loperte, A., Dolce, C., Lamiranda, L., Masini, N., and Soldovieri, F.: Integrazione di tecniche non invasive per la conoscenza strutturata degli edifici storici. Il caso studio del convento di San Francesco a Folloni (AV). Tecnologie innovative per il trattamento di beni culturali. Applicazione delle onde elettromagnetiche per diagnosi e conservazione, Edited by Bruno Bisceglia, 05/2016: chapter IV, 89-122; Aracne editrice, ISBN: 97888-548-9369-6, 2016.

Goodman, D. and Piro, S.: GPR Remote sensing in Archaeology, Springer (Ed), ISBN: 978-3-642-31856-6, ISBN: 978-3-64231857-3 (eBook), doi:10.1007/978-3-642-31857-3, Springer, Berlin, Germany, 2013.

Grajek, P. R., Schoenlinner, B., and Rebeiz, G. M.: A 24-GHz HighGain Yagi-Uda Antenna Array, 52, 1257-1261, May 2004.

Grinzato, E., Bison, P. G., and Marinetti, S.: Monitoring of ancient buildings by the thermal method, J. Cult. Herit., 3, 21-29, ISSN: 1296-2074, doi:10.1016/S1296-2074(02)01159-7, 2002.

Kadioglu, S. and Kadioglu, Y. K.: Picturing internal fractures of historical statues using ground penetrating radar method, Adv. Geosci., 24, 23-34, doi:10.5194/adgeo-24-23-2010, 2010.

Leucci, G., Masini, N., Persico, R., and Soldovieri, F.: GPR and sonic tomography for structural restoration: the case of the cathedral of Tricarico, J. Geophys. Eng., 8, S76-S92, doi:10.1088/1742-2132/8/3/S08, 2011.

Lorusso, G., Calculli, L., and Clemente, M.: La basilica cattedrale di Gravina nel tempo, Lab Edizioni, Altamura (Bari), p. 274, ISBN: 9788897796091, 2013.

Maldague, X.: Theory and Practice of Infrared Technology for Non Destructive Testing, Wiley, 2001.

Masini, N., Persico, R., and Rizzo, E.: Some Examples of GPR Prospecting for Monitoring of the Monumental Heritage, J. Geophys. Eng., 7, 190-199, doi:10.1088/1742-2132/7/2/S05, 2010.
Noon, D. A.: Stepped-Frequency Radar Design and Signal Processing Enhances Ground Penetrating Radar Performance, PhD thesis, Department of Electrical \& Computer Engineering, University of Queensland, Australia, 1996.

Persico, R.: Introduction to Ground Penetrating Radar: Inverse Scattering and data processing, Wiley, ISBN: 9781118305003, 2014

Persico, R. and Prisco, G.: A Reconfigurative Approach for SF-GPR Prospecting, IEEE Trans. On Antennas and Prop., 56, 26732680, 2008.

Persico, R., Soldovieri, F., and Pierri, R.: Convergence properties of a quadratic approcah to the inverse-scattering problem, Journal of Optical Society of America A, 19, 2424-2428, 2002.

Persico, R., Ciminale, M., and Matera, L.: A new reconfigurable stepped frequency GPR system, possibilities and issues; applications to two different Cultural Heritage Resources, Near Surf. Geophys., 12, 793-801, 2014.

Persico, R., Dei, D., Parrini, F., and Matera, L.: Mitigation of narrowband interferences by means of a reconfigurable stepped frequency GPR system, Radio Sci., 51, 1322-1331, doi:10.1002/2016RS005986, 2016.

Pieraccini, M., Noferini, L., Mecatti, D., Atzeni, C., Persico, R., and Soldovieri, F.: Advanced Processing Techniques for Stepfrequency Continuous-Wave Penetrating Radar: the Case Study of "Palazzo Vecchio" Walls (Firenze, Italy), Research on Nondestructive Evaluation, 17, 71-83, 2006.

Piscitelli, S., Rizzo, E., Cristallo, F., Lapenna, V., Crocco, L., Persico, R., and Soldovieri, F.: GPR and microwave tomography for detecting shallow cavities in the historical area of Sassi of Matera (Southern Italy), Near Surf. Geophys., 5, 275-284, doi:10.3997/1873-0604.2007009, 2007.

Sambuelli, L., Bohm, G., Capizzi, P., Cardarelli, E., and Cosentino, P.: Comparison between GPR measurements and ultrasonic tomography with different inversion algorithms: an application to the base of an ancient Egyptian sculpture, J. Geophys. Eng., 8, S106 pp., doi:10.1088/1742-2132/8/3/S10, 2011.

Sandmeier, K. J.: Reflexw 6.0 Manual Sandmeier Software ZipserStrabe1 D-76227 Karlsruhe, Germany, 2011.

Utsi, E.: The Shrine of Edward the Confessor: a Study in MultiFrequency GPR Investigation. Proc. of XIII International Conference on Ground Penetrating Radar, Lecce, 21-25 June 2010, IEEE, ISBN: 978-1-4244-4605-6, 2010. 